BMJ Open

Respiratory

Research

\title{
Home-based pulmonary rehabilitation early after hospitalisation in COPD (early HomeBase): protocol for a randomised controlled trial
}

Narelle S Cox, ${ }^{1,2}$ Aroub Lahham, ${ }^{1}$ Christine F McDonald, ${ }^{2,3,4}$ Ajay Mahal, ${ }^{5}$ Paul O'Halloran, ${ }^{6}$ Graham Hepworth, ${ }^{7}$ Lissa Spencer, ${ }^{8}$ Renae J McNamara, ${ }^{9}$ Janet Bondarenko, ${ }^{1,10}$ Heather Macdonald, ${ }^{11}$ Samantha Gavin, ${ }^{12}$ Angela T Burge,${ }^{1,2,10}$ Caitlin Le Maitre, ${ }^{10}$ Cade Ringin, ${ }^{1,10}$ Elizabeth Webb, ${ }^{1,10}$ Amanda Nichols, ${ }^{1}$ Ling-Ling Tsai, ${ }^{1,9,13}$ Nia Luxton (D) , ${ }^{1}$ Stephanie van Hilten, ${ }^{1}$ Mary Santos, ${ }^{9}$ Hayley Crute, ${ }^{11}$ Megan Byrne, ${ }^{11}$ Helen Boursinos, ${ }^{1}$ Jennifer Broe, ${ }^{1}$ Monique Corbett, ${ }^{1,10}$ Tunya Marceau, ${ }^{1,10}$ Brooke Warrick, ${ }^{1,11}$ Claire Boote, ${ }^{10}$ Joanna Melinz, ${ }^{12}$ Anne E Holland ${ }^{1,10}$

To cite: Cox NS, Lahham A, McDonald CF, et al. Home-based pulmonary rehabilitation early after hospitalisation in COPD (early HomeBase): protocol for a randomised controlled trial. BMJ Open Resp Res 2021;8:e001107. doi:10.1136/ bmjresp-2021-001107

- Additional supplemental material is published online only. To view, please visit the journal online (http://dx.doi. org/10.1136/bmjresp-2021001107).

Received 14 September 2021 Accepted 5 November 2021

Check for updates

(C) Author(s) (or their employer(s)) 2021. Re-use permitted under CC BY-NC. No commercial re-use. See rights and permissions. Published by BMJ.

For numbered affiliations see end of article.

Correspondence to Dr Narelle S Cox; Narelle.Cox@monash.edu

\section{ABSTRACT}

Introduction Chronic obstructive pulmonary disease (COPD) is characterised by exacerbations of respiratory disease, frequently requiring hospital admission.

Pulmonary rehabilitation can reduce the likelihood of future hospitalisation, but programme uptake is poor. This study aims to compare hospital readmission rates, clinical outcomes and costs between people with COPD who undertake a home-based programme of pulmonary rehabilitation commenced early (within 2 weeks) of hospital discharge with usual care.

Methods A multisite randomised controlled trial, powered for superiority, will be conducted in Australia. Eligible patients admitted to one of the participating sites for an exacerbation of COPD will be invited to participate. Participants will be randomised 1:1. Intervention group participants will undertake an 8-week programme of home-based pulmonary rehabilitation commencing within 2 weeks of hospital discharge. Control group participants will receive usual care and a weekly phone call for attention control. Outcomes will be measured by a blinded assessor at baseline, after the intervention (week 9-10 posthospital discharge), and at 12 months follow-up. The primary outcome is hospital readmission at 12 months followup.

Ethics and dissemination Human Research Ethics approval for all sites provided by Alfred Health (Project 51216). Findings will be published in peer-reviewed journals, conferences and lay publications. Trial registration number ACTRN12619001122145.

\section{INTRODUCTION}

People with chronic obstructive pulmonary disease (COPD) experience a progressive decline in lung function, reduced exercise tolerance, marked breathlessness and multiple comorbidities. ${ }^{1}$ Recurrent bouts of acute deterioration in symptoms are common in COPD, and such exacerbations ${ }^{2}$ commonly require hospital admission for treatment and management. Hospitalisation for an exacerbation of COPD is associated with increased morbidity, readmission, resource utilisation and mortality. ${ }^{3}$ Additionally, COPD exacerbations reduce both quality of life and physical function, which may not spontaneously recover. ${ }^{13}$ For people with COPD avoiding exacerbations, and resultant hospitalisations are outcomes of key importance. ${ }^{4}$

Pulmonary rehabilitation, a comprehensive programme including exercise training and self-management education, ${ }^{5}$ is an established management strategy for people with COPD recommended in guidelines. ${ }^{67}$ Systematic reviews with meta-analyses and large cohort studies have both demonstrated that completion of pulmonary rehabilitation reduces future exacerbations, need for hospitalisation and hospital length of stay. ${ }^{8-10}$ The reduced likelihood of hospital admission is particularly evident when pulmonary rehabilitation is undertaken following an exacerbation (pooled OR $0.44,95 \%$ CI 0.21 to 0.91 ). ${ }^{1}$ However, following hospitalisation for an exacerbation, fewer than $10 \%$ of people with COPD are referred to outpatient pulmonary rehabilitation on hospital discharge ${ }^{11}$ and fewer than $3 \%$ attend outpatient pulmonary rehabilitation in the year following hospital discharge. ${ }^{12}$ Low rates of pulmonary rehabilitation referral are contributed to by limited knowledge and experience of pulmonary rehabilitation by healthcare professionals. ${ }^{1314}$ 
Poor referral rates are further compounded by key patientrelated barriers to attendance at outpatient pulmonary rehabilitation programmes including limited understanding of programme requirements and benefits, and difficulties associated with travel and transport. ${ }^{1415}$

Despite evidence and growing interest in pulmonary rehabilitation following an exacerbation, ${ }^{16}$ previous studies have had challenges in terms of participant recruitment and retention, ${ }^{1718}$ had variable clinical efficacy, ${ }^{11}{ }^{19-23}$ and been heterogeneous in regard to timing of rehabilitation commencement, training duration and intensity and length of follow-up period. ${ }^{1}$ When to commence pulmonary rehabilitation following an exacerbation remains a key issue. A randomised controlled trial (RCT) with 320 participants hospitalised for an exacerbation of COPD who were allocated to either very early rehabilitation (commenced within 48 hours of admission) or to usual care reported significantly increased mortality at 1 year in the very early rehabilitation group (OR 1.74, 95\% CI 1.05 to 2.88$).{ }^{19}$ The reasons for increased mortality are not known, but a meta-analysis of rehabilitation interventions post exacerbation suggests the timing of intervention delivery is crucial, with pooled data for studies that commenced pulmonary rehabilitation in the (very) early inpatient period demonstrating increased odds of death (OR 1.74, 95\% CI 1.07 to 2.84), when compared with those studies that waited to commence rehabilitation in the period following hospital discharge (OR $0.25,95 \% \mathrm{CI}$ 0.08 to 0.75$){ }^{24} \mathrm{~A}$ rehabilitation delivery model that is acceptable to patients in the period following hospital discharge, efficacious and amenable to implementation in clinical practice is still to be elucidated.

Alternative models of pulmonary rehabilitation delivery that overcome patient barriers to attendance at outpatient programmes, have the potential to improve rates of rehabilitation completion following an exacerbation and reduce the need for subsequent hospitalisation. A recent UK national audit reported significant reductions in hospital admissions and length of stay for people who complete pulmonary rehabilitation vs noncompleters (admissions: $13 \%$ vs 27\%; length of stay: 3 days vs 7.2 days; both $\mathrm{p}<0.001) .^{25}$ Previously, we have demonstrated higher programme completion rates with a home-based model of pulmonary rehabilitation when compared with traditional outpatient pulmonary rehabilitation in a randomised controlled equivalence trial in people with stable COPD (91\% vs $49 \%$ completion). ${ }^{26}$ Equivalent clinical outcomes, at similar costs, were also achieved. ${ }^{26}$ In a small feasibility study the same homebased model of pulmonary rehabilitation was found to be satisfactory to people with COPD following hospitalisation for an exacerbation and achieved clinically meaningful improvement in quality of life and functional exercise capacity. ${ }^{27}$ Although programme uptake was modest, $80 \%$ of participants who commenced the homebased pulmonary rehabilitation programme went on to complete. ${ }^{27}$ Whether this translates into reduced need for future hospitalisation is unknown.
The aims of this study are to compare a home-based programme of pulmonary rehabilitation, delivered by telephone and commenced within 2 weeks after hospital discharge, to usual care, in people with COPD following hospitalisation for an exacerbation. In particular we aim to determine: (1) hospital readmission rates; (2) clinical outcomes and (3) costs. We hypothesise that, when compared with usual care, a home-based programme of pulmonary rehabilitation commenced early (within 2 weeks) following hospital discharge for an exacerbation of COPD will: (1) reduce hospital admissions; (2) produce clinically meaningful improvements in symptoms, health-related quality of life and exercise capacity which are greater than those seen in usual care and (3) be more cost-effective, from a societal perspective.

\section{METHODS AND ANALYSIS \\ Design}

A randomised, controlled, assessor-blinded trial, powered for superiority, will be conducted at three metropolitan and two regional centres, across multiple states, in Australia. The trial was registered prospectively at www. anzctr.org.au (ACTRN 12619001122145) on August 12 2019 , including details of trial sites. Study procedures are illustrated in figure 1.

\section{Participants}

Individuals admitted to hospital for an exacerbation of COPD at one of the participating sites will be invited to participate. Inclusion and exclusion criteria are detailed in table 1.

A clinician or researcher will provide written and verbal information to all eligible participants during their hospital stay. Visual information using a video is also available. Written informed consent will be provided by all individuals who agree to participate. Trial participation will not alter routine COPD management, nor management of any other health condition.

\section{Recruitment and randomisation}

Following informed consent, participants will be randomly allocated (1:1) to either the intervention (home-based pulmonary rehabilitation commencing early (within 2 weeks) following hospital discharge, ie, early HomeBase), or to a control group. An independent, external, service (https://randomisation.griffith.edu.au/) will be used to generate and conceal the randomisation sequence from investigators. The randomisation scheme will be computer generated, using permuted blocks and stratified for (1) site of recruitment; as well as (2) disease severity (forced expiratory volume in $1 \mathrm{~s}$ (FEV1) $\geq 50 \%$ predicted and $<50 \%$ predicted) and (3) age $(\geq 75 \mathrm{vs}<75$ years $)-$ noting both disease and age are powerful predictors of future hospitalisation and mortality in COPD ${ }^{28}$ Randomisation will take place following completion of the baseline assessment. Due to the nature of the intervention 


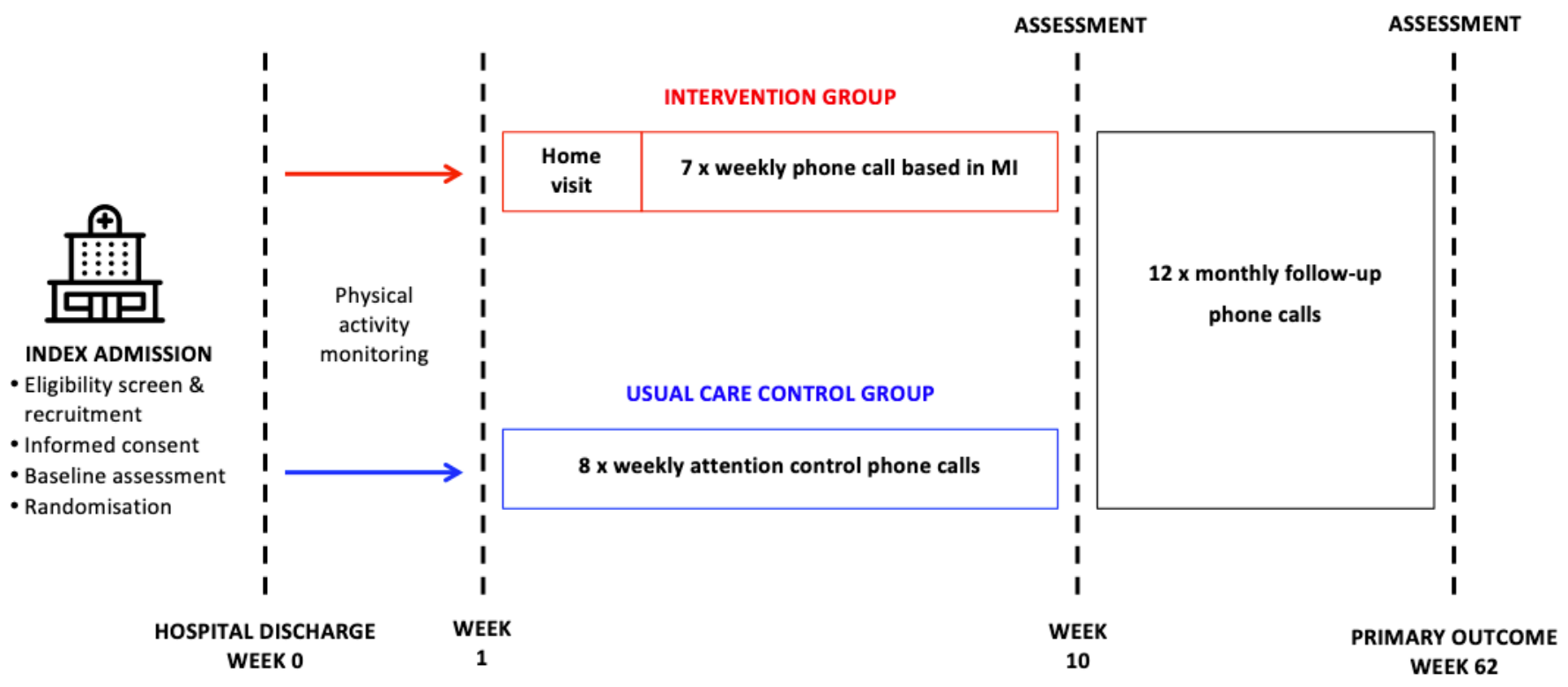

Figure 1 Study procedures. MI, motivational interviewing.

(exercise rehabilitation) it will not be possible to blind participants to the intervention, however, all outcome measures will be collected by an independent assessor blind to group allocation. Participant flow through the trial will be reported according to the recommendations of the Consolidated Standard of Reporting Trials (CONSORT and CONSERVE-CONSORT). ${ }^{29} 30$

\section{Interventions}

\section{Usual care}

All participants will receive usual care, including medical management for their exacerbation based on COPD-X guidelines $^{6}$ (medications as indicated, controlled oxygen therapy and ventilatory assistance as required) and physiotherapy, commencing on the first day of admission.

\section{Control group}

In addition to usual care, participants randomised to the control group will receive a weekly telephone call for the period of the intervention ( 8 weeks). Telephone calls will

\begin{tabular}{|c|c|}
\hline Inclusion criteria & Exclusion criteria \\
\hline Diagnosis of COPD* & Life expectancy $<6$ months ${ }^{50}$ \\
\hline $\begin{array}{l}\text { Admitted to hospital for an } \\
\text { exacerbation of COPD }\end{array}$ & $\begin{array}{l}\text { Comorbidities that preclude } \\
\text { exercise trainingt }\end{array}$ \\
\hline Age $\geq 40$ years & $\begin{array}{l}\text { Inability to provide informed } \\
\text { consent }\end{array}$ \\
\hline
\end{tabular}

*All GOLD stages considered for eligilibity.

†Comorbidities may include, but are not limited to, neurological or musculoskeletal impairment; acute unstable cardiac disease.

COPD, chronic obstructive pulmonary disease . not include any health information or advice on physical activity or exercise and are designed to serve as attention control. A standardised telephone call record sheet will be used to record participant comments in response to how they are feeling and whether they have visited the doctor, hospital or emergency department in the previous week. In line with guideline recommendations for management of an acute exacerbation of $\mathrm{COPD},{ }^{6}$ participants in the control group may be referred to traditional outpatient (centre-based) pulmonary rehabilitation after discharge from hospital. The number of control participants who take up such a referral will be documented.

\section{Intervention group: home-based pulmonary rehabilitation} commenced early after hospitalisation (early HomeBase)

Alongside usual care, participants in the intervention group will undertake an 8-week home-based, pulmonary rehabilitation programme commenced within 2 weeks of hospital discharge. Early home-based pulmonary rehabilitation will be delivered according to our previously published protocol in stable COPD. ${ }^{26}$ The nature of the home-based intervention, that is, being delivered by telephone, conforms with the definitions of both telehealth and telerehabilitation being healthcare (specifically rehabilitation) provided at a distance through the use of telecommunication (or virtual) technology. ${ }^{31}$

\section{Home visit}

According to our previously published model,${ }^{26}$ the programme will commence with one home-visit by a physiotherapist to establish exercise goals and safety, and supervise the first exercise session. Also included in the home-visit will be components of the self-management programme that cannot be adequately covered by subsequent telephone calls (eg, review of inhaler technique) ${ }^{26}$ 


\section{Self-monitored exercise training}

Participants will undertake an aerobic and resistance training programme, with intensity of training individualised according to symptoms to achieve a Borg-CR10 breathlessness rating of 3-4 with exercise. ${ }^{32}$ For aerobic training, a walking distance goal will be set, with distance assessed using pedometers. Where participants have interest and access to alternative aerobic training modalities, for example, cycling or swimming, these may undertaken in addition to or instead of walking training. The target will be $30 \mathrm{~min}$ of aerobic exercise per day, which may be undertaken in multiple shorter periods. At least five sessions of aerobic training per week, will be encouraged. Resistance training will use functional activities and equipment that are readily accessible in the home environment, such as sit-to-stand from a dining chair and water bottles for upper limb weights. Participants will record details of their exercise training in a home exercise diary.

The initial home-visit will be followed by seven onceweekly telephone calls from a pulmonary rehabilitation clinician, using a motivational interviewing approach. Clinicians delivering intervention phone calls will undertake specialised motivational interviewing training, and receive ongoing support and training from an expert motivational interviewing practitioner, to ensure competency. During the telephone calls the pulmonary rehabilitation clinician will (1) review symptoms and the home diary; (2) progress the exercise prescription through discussion of exercise goals, with goals recorded in the home diary and (3) facilitate self management of exacerbations and deliver self-management education via scripted telephone modules. These previously developed, ${ }^{26}$ structured telephone modules will be used to explore and build motivation for exercise participation, then move towards commitment and action. ${ }^{33}$

\section{Self-management education}

To address international guidelines, ${ }^{7}$ participants will be provided with a selection of topics related to COPD selfcare and encouraged to choose a topic of relevance to them for discussion each week during their telephone call with the clinician. ${ }^{34}$ Education on how to manage an acute exacerbation of COPD and the importance of long-term exercise maintenance will be a focus of selfmanagement training for all participants. The aim is to improve self-efficacy by increasing the knowledge and skills required to optimally manage COPD. This approach requires goal setting, problem solving, decision making and taking action based on a predefined plan. ${ }^{34}$ Other positive adaptive behaviours that may be facilitated during self-management training include medication adherence, smoking cessation and changing nutritional habits. Health goals will be documented in the home exercise diary and reviewed weekly. Participants will also receive self-management education resources from Lung Foundation Australia. These resources have been developed to enable people with COPD to undertake the educational component of pulmonary rehabilitation from the comfort of their own home and include a book (Better Living with COPD: A Patient Guide(third Ed) $)^{35}$ and access to the C.O.P.E (COPD Online Patient Education) programme.

\section{Ongoing exercise participation}

At the completion of the rehabilitation period participants in the intervention group will be offered the opportunity to join a supervised exercise maintenance programme to promote ongoing exercise adherence, in line with national standards for postpulmonary rehabilitation care. ${ }^{36}$ Acceptance of a referral will be documented. Participants in the intervention group will be precluded from attending standard outpatient pulmonary rehabilitation only during the intervention period (ie, weeks 0-10).

\section{Safety}

Prior to commencing each unsupervised exercise session at home, intervention group participants will be asked to complete a checklist to ensure clinical stability for exercise (see online supplemental material). If the participant has symptoms of an exacerbation, the participant will be instructed to refrain from exercise and contact one of the researchers. If discussion confirms symptoms are likely to be indicative of a moderate exacerbation then the participant will be instructed not to exercise and to seek medical attention. If there is evidence of a severe exacerbation, an ambulance will be called. This is consistent with procedures that would be undertaken in a centre-based pulmonary rehabilitation programme and were successfully used previously. ${ }^{26}$

To further monitor for safety, all participants (intervention and control groups) will be asked about emergency department presentation and/or hospital admission at their weekly phone call during the intervention period, and during their monthly phone call during the 12-month follow-up period. In the event a participant is unable to be reached by telephone for a scheduled call, a phonecall will be made to the next-of-kin to check on participant well-being.

\section{Outcomes}

Baseline participant characteristics including age, sex, lung function, frailty score, ${ }^{37}$ medications and hospitalisations will be collected from the participants and the medical record during the index hospital admission. Clinical outcome measures (table 2) will be assessed at baseline, at the end of the intervention (week 9-10 posthospital discharge), and at 12 months follow-up. All outcome measures will be collected by a researcher blind to group allocation. Participants will be asked to attend the hospital of recruitment for the end-intervention and follow-up assessments to standardise the administration 
Table 2 Clinical outcome measures and assessment time points

\begin{tabular}{|c|c|c|c|c|}
\hline Outcome & Measure & Description & $\begin{array}{l}\text { Assessment time } \\
\text { point(s) }\end{array}$ & \\
\hline $\begin{array}{l}\text { Readmission } \\
\text { to hospital }\end{array}$ & $\begin{array}{l}\text { Hospital } \\
\text { admission }\end{array}$ & $\begin{array}{l}\text { Determined using hospital medical records and } \\
\text { cross-checked with patient self-report via monthly } \\
\text { telephone calls }\end{array}$ & 12 months & $\begin{array}{l}\text { Primary } \\
\text { outcome }\end{array}$ \\
\hline $\begin{array}{l}\text { Exercise } \\
\text { capacity }\end{array}$ & 1STS & $\begin{array}{l}\text { A valid, reliable and responsive test to assess } \\
\text { exercise capacity in people with COPD. }{ }^{51} \text { The test } \\
\text { will be conducted twice and the best result will be } \\
\text { recorded. }\end{array}$ & $\begin{array}{l}\text { Baseline } \\
\text { End-intervention } \\
12 \text { months }\end{array}$ & $\begin{array}{l}\text { Secondary } \\
\text { outcomes }\end{array}$ \\
\hline Self-efficacy & PRAISE & $\begin{array}{l}\text { A reliable and sensitive measure of self-efficacy } \\
\text { for patients attending pulmonary rehabilitation. }{ }^{52}\end{array}$ & $\begin{array}{l}\text { Baseline } \\
\text { End-intervention } \\
12 \text { months }\end{array}$ & \\
\hline Dyspnoea & mMRC & $\begin{array}{l}\text { The mMRC provides a measure of functional } \\
\text { breathlessness on a scale from } 0 \text { to } 4 \text {, where } \\
0 \text { indicates no restriction to activities due to } \\
\text { breathlessness and } 4 \text { the greatest impairment. }\end{array}$ & $\begin{array}{l}\text { Baseline } \\
\text { End-intervention } \\
12 \text { months }\end{array}$ & \\
\hline $\begin{array}{l}\text { Anxiety/ } \\
\text { Depression }\end{array}$ & HADS & $\begin{array}{l}\text { Anxiety and depression are common } \\
\text { comorbidities in people with COPD and can be } \\
\text { ameliorated with pulmonary rehabilitation, }{ }^{34} \text { and } \\
\text { will be evaluated using the HADS. }{ }^{54}\end{array}$ & $\begin{array}{l}\text { Baseline } \\
\text { End-intervention } \\
12 \text { months }\end{array}$ & \\
\hline \multirow[t]{2}{*}{$\begin{array}{l}\text { Health-related } \\
\text { QoL }\end{array}$} & CRDQ & $\begin{array}{l}\text { A disease-specific measure of health-related } \\
\text { QoL. }{ }^{55} \text { Is a valid measure of health-related QoL in } \\
\text { people with COPD and responsive to change with } \\
\text { pulmonary rehabilitation. }{ }^{56}\end{array}$ & $\begin{array}{l}\text { Baseline } \\
\text { End-intervention } \\
12 \text { months }\end{array}$ & \\
\hline & EQ-5D-5L & $\begin{array}{l}\text { The EQ-5D-5L is a validated generic QoL measure } \\
\text { which is used to estimate health benefits in terms } \\
\text { of QALYs, and is recommended for economic } \\
\text { analyses. } 57\end{array}$ & $\begin{array}{l}\text { Baseline } \\
\text { End-intervention } \\
12 \text { months }\end{array}$ & \\
\hline $\begin{array}{l}\text { Physical } \\
\text { activity }\end{array}$ & $\begin{array}{l}\text { Accelerometry } \\
\text { (Actigraph } \\
\text { wGT3x-BT; } \\
\text { Actigraph, } \\
\text { Pensacola, FL } \\
32502 \text { USA) }\end{array}$ & $\begin{array}{l}\text { Physical activity levels are an important measure } \\
\text { of behaviour change following pulmonary } \\
\text { rehabilitation. Using a hip-worn tri-axial } \\
\text { accelerometer, validated for the assessment of } \\
\text { free-living activity in people with COPD, }{ }^{58} \text { data } \\
\text { relating to time spent in different physical activity } \\
\text { intensities and sedentary time will be collected } \\
\text { and stored as raw acceleration in g units (m/s2). }\end{array}$ & $\begin{array}{l}\text { Seven days of monitoring } \\
\text { to accurately capture all } \\
\text { activity intensities }{ }^{59} \text { at: } \\
\text { Baseline (immediately } \\
\text { following hospital } \\
\text { discharge) } \\
\text { End intervention } \\
12 \text { months }\end{array}$ & \\
\hline
\end{tabular}

COPD, chronic obstructive pulmonary disease; CRDQ, Chronic Respiratory Disease Questionnaire; HADS, Hospital Anxiety and Depression Scale; mMRC, Modified Medical Research Council; PRAISE, Pulmonary Rehabilitation Adapted Index of Self Efficacy; QALY, quality-adjusted life-years; QoL, quality of life; 1STS, 1-minute sit-to-stand.

of assessments across groups. The proportion of participants in both groups who take up an offer of centre-based pulmonary rehabilitation at any time in the study period will be recorded. The number of rehabilitation sessions attended (intervention and/or follow-up period) will be documented, with programme completion defined a priori as undertaking a minimum of $70 \%$ of planned sessions in any one course of pulmonary rehabilitation. ${ }^{38}$

The primary outcome is hospital readmission at 12 months. Readmission to hospital is a powerful predictor of poor outcomes in people with COPD. ${ }^{39}$ Hospital readmissions within the 12-month follow-up period will be determined using hospital medical records and crosschecked with patient self-report via monthly telephone calls. ${ }^{2640}$

\section{Economic evaluation}

Economic evaluation will take a societal perspective, ${ }^{41}$ and include an incremental cost-effective analysis, as described previously. ${ }^{40}$ Costs incurred by all stakeholders, including out-of-pocket costs to participants for healthcare services and the cost of delivering the intervention, will be included in the evaluation. Healthcare utilisation by participants, including visits to health professionals and any hospitalisations, will be recorded in a diary for 12 months following the intervention period. Participants will also be telephoned monthly for 12 months to facilitate diary completion and to capture patient-reported information on hospitalisation episodes. Hospitalisation and use of other hospital services will be confirmed by medical record audit at the completion of 12 months 
follow-up. Health benefits in terms of quality-adjusted life-years will be estimated from the utility index calculated by applying a social tariff to the EQ-5D-5 $\mathrm{L}^{42}$ and compared between groups. A separate assessment of cost-effectiveness will be undertaken by comparing the number of hospital admissions per participant in the 12-month follow-up period to the incremental cost of averting an additional hospital admission.

\section{Statistical analysis}

\section{Sample size}

To detect a difference in hospital readmission between the intervention group (early home-based pulmonary rehabilitation) and control group at 12 months follow-up, a total of 132 participants (66 participants in each group) will be required to detect a reduction in readmission rate to $30 \%$ with $80 \%$ power and a significance level of $\mathrm{p}<0.05$. This assumes a rate of readmission of $57 \%^{22}$ in the control group. An additional 34 participants will be recruited to allow for $20 \%$ drop-out. This is a conservative estimate for attrition, with our previous studies of homebased pulmonary rehabilitation demonstrating around $15 \%$ drop-out rate ${ }^{26}$; we will, therefore, randomise a total of 166 participants.

\section{Data analysis plan}

Analysis will use intention-to-treat principles, with inclusion of all randomised participants regardless of programme completion. Protocol violations will not constitute grounds for withdrawal. A participant will be considered to have withdrawn from the study if consent is revoked. Where this occurs, no further assessments will be performed, however, participants will be informed that unless permission is expressly denied data collected up to the time of withdrawal will form part of the study results. Withdrawn participants will not be replaced. For the primary hypothesis (hospital readmission), the relative risk in the intervention group compared with the usual care control group will be reported with a $95 \%$ CI. Secondary continuous outcomes will be examined using linear mixed models with fixed effects for group, time and group $\times$ time interaction, in addition to disease severity and age. Site will be included in the model as a random effect. A per protocol analysis will be undertaken including those who complete the home-based pulmonary programme ( $\geq 70 \%$ of sessions attended). The proportion of participants in both groups who attend at least $70 \%$ of a pulmonary rehabilitation programme (home-based pulmonary rehabilitation intervention and/or outpatient pulmonary rehabilitation) in the study period (from hospital discharge to 12 months follow-up) will be compared using logistic regression. We will explore time to readmission using Cox regression, controlling for programme completion.
Data management

A purpose-built on-line database (www.adeptrs.com), with encryption and password protection, will be used to store electronic data. No identifying information will be stored in the online database. Hard copy original data collection forms will be stored in a locked filing cabinet within a locked office at the site of participant recruitment.

\section{Monitoring}

A multidisciplinary data safety and monitoring committee who, collectively, have experience in the management of patients with COPD, the delivery of pulmonary rehabilitation and the conduct and monitoring of RCTs, will meet a minimum of twice yearly. The Committee will include a biostatistician, and be chaired by a respiratory physician who is independent of the study team and the trial sites. The data safety monitoring committee will review data in a blinded fashion.

\section{Dissemination}

It is intended study findings will be disseminated via publication in peer-reviewed journals and presentation at conferences. A plain-English summary will be sent to participants at the conclusion of the trial, and results conveyed to people with COPD through lay publications and seminars.

\section{DISCUSSION}

Pulmonary rehabilitation is a proven treatment for people with $\mathrm{COPD}^{8}$ yet is widely underused, particularly following an episode of hospitalisation for an exacerbation. ${ }^{11}$ Despite guideline recommendations for referral to pulmonary rehabilitation following exacerbation, ${ }^{7}$ there is a widespread lack of implementation of outpatient pulmonary rehabilitation after hospitalisation. In addition, doubts around timing of commencement of rehabilitation programmes following exacerbation have led to international calls for research evaluating alternative models of pulmonary rehabilitation that offer flexibility in terms of setting, type of training and timing of intervention delivery following hospitalisation. ${ }^{43} 44$

COPD is the most prevalent respiratory disease worldwide $^{45}$ and is expected to become the leading cause of mortality, globally, within the next two decades. ${ }^{46}$ The economic burden of COPD is considerable, at both a patientand health-system level, ${ }^{47}$ with nearly three-quarters of healthcare costs associated with COPD attributable to exacerbations requiring hospitalisation. ${ }^{48}$ Pulmonary rehabilitation is a relatively low-cost, cost-effective treatment strategy ${ }^{49}$ that can reduce the likelihood of future need for hospitalisation due to exacerbation. ${ }^{8}$ To impact on rehospitalisation rates more people with COPD need to complete pulmonary rehabilitation. ${ }^{25}$ The period early posthospitalisation appears critical to reducing the likelihood of future hospitalisation, however key barriers to 
attending outpatient pulmonary rehabilitation must be overcome in order to achieve this outcome. The American Thoracic Society/European Respiratory Society Pulmonary Rehabilitation Policy Statement has identified as key research priorities the investigation of models of pulmonary rehabilitation delivery that improve access and uptake for patients; establish the cost-effectiveness of programmes, and address patient barriers to programme access, enrolment and completion. ${ }^{43}$ The randomised, controlled, superiority, trial described here, with embedded economic analysis, uses an alternative (homebased) model of pulmonary rehabilitation delivery, rigorously tested in stable patients, ${ }^{26}$ to address these research priorities and outcomes of importance to patients and the health system.

If this study demonstrates that home-based pulmonary rehabilitation, commenced within 2 weeks of hospital discharge, is effective at reducing hospital admissions and improving rehabilitation completion rates it will offer an accessible model of pulmonary rehabilitation, requiring minimal resources, for the period following COPD exacerbation. This low-cost home-based model of pulmonary rehabilitation is designed for rapid uptake into clinical practice on a global scale, including in locations where access is particularly limited, such as lowresource settings.

\section{Patient and public involvement}

The experiences of patients from our previous trials of home-based pulmonary rehabilitation ${ }^{26}$ and our pilot study of early home-based pulmonary rehabilitation ${ }^{27}$ informed the development of the protocol including timing of recruitment and assessments and intervention resources. People living with chronic lung disease provided patient review of intervention resources through the Lung Foundation Australia patient advocacy groups. Hospitalisation was selected as the primary outcome of the trial as it is an outcome of key importance to people with COPD. ${ }^{4}$

\section{Trial status}

Recruitment to the trial commenced at a single site (Alfred Health, Melbourne) in January 2020, with the remaining sites scheduled to commence recruitment by April 20, 2020. Due to delays associated with the COVID-19 pandemic, recruitment at all sites had commenced by November 2020. It is estimated the recruitment goal will be met by December 2023 .

\footnotetext{
Author affiliations

${ }^{1}$ Respiratory Research@Alfred, Department of Immunology \& Pathology, Monash University, Melbourne, Victoria, Australia

${ }^{2}$ Institute for Breathing and Sleep, Melbourne, Victoria, Australia

${ }^{3}$ Respiratory and Sleep Medicine, Austin Health, Heidelberg, Victoria, Australia

${ }^{4}$ Faculty of Medicine, University of Melbourne, Melbourne, Victoria, Australia

${ }^{5}$ Melbourne School of Population and Global Health, University of Melbourne, Melbourne, Victoria, Australia
}

${ }^{6}$ School of Psychology and Public Health, La Trobe University, Melbourne, Victoria, Australia

${ }^{7}$ Statistical Consulting Centre, University of Melbourne, Melbourne, Victoria, Australia

${ }^{8}$ Royal Prince Alfred Hospital, Camperdown, New South Wales, Australia

${ }^{9}$ Prince of Wales Hospital, Sydney, New South Wales, Australia

${ }^{10}$ Alfred Health, Melbourne, Victoria, Australia

${ }^{11}$ Wimmera Health Care Group, Horsham, Victoria, Australia

${ }^{12}$ Coffs Harbour Health Campus, Coffs Harbour, New South Wales, Australia

${ }^{13}$ Sydney Local Health District, Sydney, New South Wales, Australia

Contributors Procured funding: NSC, AEH, CFM, AM, PO'H and GH. Conceptualisation and design: NSC, AEH, CFM, AM, PO'H and GH. Drafting protocol manuscript: NSC and AEH. Critical review of protocol manuscript: AEH, CFM, AM, PO'H, GH, AL, LS, RJM, JB, HM, SG, ATB, CLM, CR, EW, AN, L-LT, NL, SvH, MS, HC, $M B, B W, H B, J B, T M, M C, C B$ and JM.

Funding This work is supported by a National Health and Medical Research Council (NHMRC) project grant (GNT1157313) held by NS Cox and AE Holland.

Competing interests NSC and AEH: declare grant funding from the NHMRC paid to their institution for the conduct of the trial. CFM declares board roles as Chair COPD National Program, Lung Foundation Australia; and Medical Director Institute for Breathing and Sleep. For all other authors, no competing interests are declared.

Patient consent for publication Not applicable.

Ethics approval Human Research Ethics approval was provided by Alfred Health for all sites (Project ID 51216, Local reference 142/19) with local governance approvals obtained from all participating sites.

Provenance and peer review Not commissioned; externally peer reviewed.

Supplemental material This content has been supplied by the author(s). It has not been vetted by BMJ Publishing Group Limited (BMJ) and may not have been peer-reviewed. Any opinions or recommendations discussed are solely those of the author(s) and are not endorsed by BMJ. BMJ disclaims all liability and responsibility arising from any reliance placed on the content. Where the content includes any translated material, BMJ does not warrant the accuracy and reliability of the translations (including but not limited to local regulations, clinical guidelines, terminology, drug names and drug dosages), and is not responsible for any error and/or omissions arising from translation and adaptation or otherwise.

Open access This is an open access article distributed in accordance with the Creative Commons Attribution Non Commercial (CC BY-NC 4.0) license, which permits others to distribute, remix, adapt, build upon this work non-commercially, and license their derivative works on different terms, provided the original work is properly cited, appropriate credit is given, any changes made indicated, and the use is non-commercial. See: http://creativecommons.org/licenses/by-nc/4.0/.

ORCID ID

Nia Luxton http://orcid.org/0000-0003-2789-2275

\section{REFERENCES}

1 Puhan MA, Gimeno-Santos E, Cates CJ, et al. Pulmonary rehabilitation following exacerbations of chronic obstructive pulmonary disease. Cochrane Database Syst Rev 2016;12:CD005305.

2 Kim V, Aaron SD. What is a COPD exacerbation? current definitions, pitfalls, challenges and opportunities for improvement. Eur Respir J 2018;52:1801261.

3 Piquet J, Chavaillon J-M, David P, et al. High-Risk patients following hospitalisation for an acute exacerbation of COPD. Eur Respir $J$ 2013;42:946-55.

4 Zhang Y, Morgan RL, Alonso-Coello P, et al. A systematic review of how patients value COPD outcomes. Eur Respir J 2018;52. doi:10.1183/13993003.00222-2018. [Epub ahead of print: 1907 2018].

5 Holland AE, Cox NS, Houchen-Wolloff L, et al. Defining modern pulmonary rehabilitation. An official American thoracic Society workshop report. Ann Am Thorac Soc 2021;18:e12-29.

6 Yang IA, Brown JL, George J, et al. COPD-X Australian and New Zealand guidelines for the diagnosis and management of chronic obstructive pulmonary disease: 2017 update. Med J Aust 2017;207:436-42.

7 Global strategy for the diagnosis, management and prevention of COPD, 2021. Available: http://goldcopd.org 
8 McCarthy B, Casey D, Devane D, et al. Pulmonary rehabilitation for chronic obstructive pulmonary disease. Cochrane Database Syst Rev 2015:CD003793.

9 Ryrsø CK, Godtfredsen NS, Kofod LM, et al. Lower mortality after early supervised pulmonary rehabilitation following COPDexacerbations: a systematic review and meta-analysis. BMC Pulm Med 2018;18:154.

10 Stefan MS, Pekow PS, Priya A, et al. Association between initiation of pulmonary rehabilitation and rehospitalizations in patients hospitalized with chronic obstructive pulmonary disease. $\mathrm{Am} \mathrm{J}$ Respir Crit Care Med 2021:204:1015-23.

11 Jones SE, Green SA, Clark AL, et al. Pulmonary rehabilitation following hospitalisation for acute exacerbation of COPD: referrals, uptake and adherence. Thorax 2014;69:181-2.

12 Spitzer KA, Stefan MS, Priya A. Participation in pulmonary rehabilitation following hospitalization for COPD among Medicare beneficiaries. Ann Am Thorac Soc 2019;16:99-106.

13 Milner SC, Boruff JT, Beaurepaire C, et al. Rate of, and barriers and enablers to, pulmonary rehabilitation referral in COPD: a systematic scoping review. Respir Med 2018;137:103-14.

14 Cox NS, Oliveira CC, Lahham A, et al. Pulmonary rehabilitation referral and participation are commonly influenced by environment, knowledge, and beliefs about consequences: a systematic review using the theoretical domains framework. J Physiother 2017;63:84-93.

15 Keating A, Lee AL, Holland AE. Lack of perceived benefit and inadequate transport influence uptake and completion of pulmonary rehabilitation in people with chronic obstructive pulmonary disease: a qualitative study. J Physiother 2011;57:183-90.

16 Man WD-C, Puhan MA, Harrison SL, et al. Pulmonary rehabilitation and severe exacerbations of COPD: solution or white elephant? ERJ Open Res 2015;1:00050-2015.

17 Revitt O, Sewell L, Singh S. Early versus delayed pulmonary rehabilitation: A randomized controlled trial - Can we do it? Chron Respir Dis 2018:15:323-6.

18 Milner SC, Bourbeau J, Ahmed S, et al. Improving acceptance and uptake of pulmonary rehabilitation after acute exacerbation of COPD: Acceptability, feasibility, and safety of a PR "taster" session delivered before hospital discharge. Chron Respir Dis 2019;16:1479973119872517.

19 Greening NJ, Williams JEA, Hussain SF, et al. An early rehabilitation intervention to enhance recovery during hospital admission for an exacerbation of chronic respiratory disease: randomised controlled trial. BMJ 2014;349:g4315.

20 Ko FWS, Dai DLK, Ngai J, et al. Effect of early pulmonary rehabilitation on health care utilization and health status in patients hospitalized with acute exacerbations of COPD. Respirology 2011;16:617-24.

21 Eaton T, Young P, Fergusson W, et al. Does early pulmonary rehabilitation reduce acute health-care utilization in COPD patients admitted with an exacerbation? A randomized controlled study. Respirology 2009;14:230-8.

22 Man WD-C, Polkey Ml, Donaldson N, et al. Community pulmonary rehabilitation after hospitalisation for acute exacerbations of chronic obstructive pulmonary disease: randomised controlled study. BMJ 2004;329:1209.

23 Kjærgaard JL, Juhl CB, Lange P, et al. Early pulmonary rehabilitation after acute exacerbation of COPD: a randomised controlled trial. ERJ Open Res 2020;6:00173-2019.

24 Holland AE. Physiotherapy management of acute exacerbations of chronic obstructive pulmonary disease. J Physiother 2014:60:181-8.

25 Steiner M, McMillan V, Lowe D. Pulmonary rehabilitation: beyond breathing better. National chronic obstructive pulmonary disease (COPD) audit programme: outcomes from the clinical audit of pulmonary rehabilitation services in England 2015. London: National supplementary report, 2017.

26 Holland AE, Mahal A, Hill CJ, et al. Home-Based rehabilitation for COPD using minimal resources: a randomised, controlled equivalence trial. Thorax 2017;72:57-65.

27 Wageck B, Cox NS, Bondarenko J, et al. Early home-based pulmonary rehabilitation following acute exacerbation of COPD: a feasibility study using an action research approach. Chron Respir Dis 2020;17:1479973120949207.

28 Soler-Cataluña JJ, Martínez-García MA, Román Sánchez P, et al. Severe acute exacerbations and mortality in patients with chronic obstructive pulmonary disease. Thorax 2005;60:925-31.

29 Schulz KF, Altman DG, Moher D. Consort 2010 statement: updated guidelines for reporting parallel group randomised trials. BMC Med 2010;8:18.

30 Orkin AM, Gill PJ, Ghersi D, et al. Guidelines for reporting trial protocols and completed trials modified due to the COVID-19 pandemic and other Extenuating circumstances: the conserve 2021 statement. JAMA 2021;326:257-65.

31 Cox NS, Dal Corso S, Hansen $\mathrm{H}$, et al. Telerehabilitation for chronic respiratory disease. Cochrane Database Syst Rev 2021;1:CD013040.

32 Borg GA. Psychophysical bases of perceived exertion. Med Sci Sports Exerc 1982;14:377-81.

33 Rollnick S, Miller W, Butler C. Motivational interviewing in health care. helping patients change behaviour. New York: Guildford Press, 2008.

34 Spruit MA, Singh SJ, Garvey C, et al. An official American thoracic Society/European respiratory Society statement: key concepts and advances in pulmonary rehabilitation. Am J Respir Crit Care Med 2013:188:e13-64.

35 Queensland Health Statewide Respiratory Clinical Network and Lung Foundation Australia. Better living with chronic obstructive pulmonary disease a patient guide. 3rd ed. Queensland, 2016.

36 Alison JA, McKeough ZJ, Johnston K, et al. Australian and New Zealand pulmonary rehabilitation guidelines. Respirology 2017;22:800-19.

37 Rockwood K, Song X, MacKnight C, et al. A global clinical measure of fitness and frailty in elderly people. CMAJ 2005;173:489-95.

38 Williams MT, Lewis LK, McKeough Z, et al. Reporting of exercise attendance rates for people with chronic obstructive pulmonary disease: a systematic review. Respirology 2014;19:30-7.

39 Suissa S, Dell'Aniello S, Ernst P. Long-Term natural history of chronic obstructive pulmonary disease: severe exacerbations and mortality. Thorax 2012;67:957-63.

40 Burge AT, Holland AE, McDonald CF, et al. Home-Based pulmonary rehabilitation for COPD using minimal resources: an economic analysis. Respirology 2020;25:183-90.

41 Cazzola M, MacNee W, Martinez FJ, et al. Outcomes for COPD pharmacological trials: from lung function to biomarkers. Eur Respir J 2008;31:416-69.

42 Devlin N, Parkin D, Janssen B. Analysis of EQ-5D values. In: Devlin N, Parkin D, Janssen B, eds. Methods for analysing and reporting EQ-5D data. Cham: Springer International Publishing, 2020: $61-86$.

43 Rochester CL, Vogiatzis I, Holland AE, et al. An official American thoracic Society/European respiratory Society policy statement: enhancing implementation, use, and delivery of pulmonary rehabilitation. Am J Respir Crit Care Med 2015;192:1373-86.

44 Janaudis-Ferreira T, Harrison SL, Ahmed S, et al. Canadian consensus recommendations for a research agenda in pulmonary rehabilitation post-acute exacerbation of COPD: a meeting report. Can J Respir Crit Care Sleep Med 2021;5:43-50.

45 GBD Chronic Respiratory Disease Collaborators. Prevalence and attributable health burden of chronic respiratory diseases, 19902017: a systematic analysis for the global burden of disease study 2017. Lancet Respir Med 2020;8:585-96.

46 Quaderi SA, Hurst JR. The unmet global burden of COPD. Glob Health Epidemiol Genom 2018;3:e4.

47 Iheanacho I, Zhang S, King D, et al. Economic burden of chronic obstructive pulmonary disease (COPD): a systematic literature review. Int J Chron Obstruct Pulmon Dis 2020;15:439-60.

48 Press VG, Konetzka RT, White SR. Insights about the economic impact of chronic obstructive pulmonary disease readmissions post implementation of the hospital readmission reduction program. Curr Opin Pulm Med 2018;24:138-46.

49 Zoumot Z, Jordan S, Hopkinson NS. Emphysema: time to say farewell to therapeutic nihilism. Thorax 2014;69:973-5

50 van de Bool C, Rutten EPA, van Helvoort A, et al. A randomized clinical trial investigating the efficacy of targeted nutrition as adjunct to exercise training in COPD. J Cachexia Sarcopenia Muscle 2017:8:748-58

51 Ozalevli S, Ozden A, Itil O, et al. Comparison of the Sit-to-Stand test with 6 min walk test in patients with chronic obstructive pulmonary disease. Respir Med 2007;101:286-93.

52 Vincent E, Sewell L, Wagg K, et al. Measuring a change in selfefficacy following pulmonary rehabilitation: an evaluation of the praise tool. Chest 2011;140:1534-9.

53 Mahler DA, Rosiello RA, Harver A, et al. Comparison of clinical dyspnea ratings and psychophysical measurements of respiratory sensation in obstructive airway disease. Am Rev Respir Dis 1987:135:1229-33.

54 Bratås O, Grønning K, Forbord T. Psychometric properties of the hospital anxiety and depression scale and the general health Questionnaire-20 in COPD inpatients. Scand J Caring Sci 2014;28:413-20.

55 Guyatt GH, Berman LB, Townsend M, et al. A measure of quality of life for clinical trials in chronic lung disease. Thorax 1987;42:773-8. 
56 Williams JE, Singh SJ, Sewell L, et al. Development of a self-reported chronic respiratory questionnaire (CRQ-SR). Thorax 2001;56:954-9.

57 Nolan CM, Longworth L, Lord J, et al. The EQ-5D-5L health status questionnaire in COPD: validity, responsiveness and minimum important difference. Thorax 2016;71:493-500.
58 Rabinovich RA, Louvaris Z, Raste Y, et al. Validity of physical activity monitors during daily life in patients with COPD. Eur Respir $J$ 2013;42:1205-15.

59 Dillon CB, Fitzgerald AP, Kearney PM, et al. Number of days required to estimate habitual activity using Wrist-Worn GENEActiv Accelerometer: a cross-sectional study. PLoS One 2016;11:e0109913. 